American Journal of Applied Sciences 8 (6): 579-583, 2011

ISSN 1546-9239

(C) 2011 Science Publications

\title{
IPv6 Network Mobility Route Optimization Survey
}

\author{
Samer Sami Hassan and Rosilah Hassan \\ School of Computer Science, University Kebangsaan Bangi, \\ 43600, Malaysia
}

\begin{abstract}
Problem statement: This study describes that the Next Generation of Networks (NGN) communication will supports multiple technologies, handles the mobility of end users to move through heterogeneous access networks, with ability to connect to different networks. Where the Internet Engineer Task Force maintain (IETF) the Mobile IPv6 (MIPv6) to handles the mobility of networks (NEMO), to provide wide band and more scalable network services. One of the MIPv6 built-in features is Route Optimization (RO) to solve the inefficient route problem. Conclusion/Recommendations: The main objective of this article is to survey, classify and make a compression between the available schemes for route optimization over the last years depends on the basic criteria generated from the published articles within different network topology. This article presents the problem of suboptimal route which is further increased with increasing of nesting levels and there is no such one scheme is perfect for all network environments.
\end{abstract}

Key words: Next Generation of Networks (NGN), Mobile IP (MIP), route optimization, Mobile Network Node (MNN), Engineer Task Force maintain (IETF), IPv6 protocol, Ethernet, Mobile Binding (BU), Home Address (HoA)

\section{INTRODUCTION}

The Mobile IPv6 protocol (MIPv6) (Johnson et al., 2004) known as a layer 3 protocol which allows mobile user's services (mobile nodes) to ongoing and reachable independently on the movement of mobile nodes in the IP environment. Without supporting mobility in IPv6 protocol, the payload destined to the MNN could not be reached and delivered as far as the mobile node was change his current location away from home network. So for keeping Mobile IP provides an IP node the ability to retain the same IP address and maintain uninterrupted network and application connectivity while traveling across networks. However, this will lead to breaks transports and higher layers connection. The Mobile IP protocol allows the Mobile Network Node (MNN) to move among heterogeneous subnets without changing its Home Address (HoA). This movement is absolutely transparent to the higher layers and packets sends to this node can be routed through the network regardless of its current location. So MIPv6 witch means an "always on" IP service availability is independent of location, movement and infrastructure (Zafar et al., 2010). The Mobile IP protocol is appropriate for providing mobility through subnets of the homogeneous access media as well as across heterogeneous access media kinds (e.g., Wi-Fi, WiMax, UMTS, Ethernet). In addition to reach ability and maintaining ongoing connections, the protocol allows for optimal routing between mobile nodes and other nodes they are communicating with. Different researches directions are proposed as shown in Fig. 1 to deal with the mobility of IPv6.

Mobile IPv6: The main principles of the NEMO (Devarapalli et al., 2005) basic support protocol are the following, there are three entities defined in MIPv6; Correspondent Node (CN), Mobile Node (MNN) and Home Agent (HA), also two Access Routers (AR): Next Access Router (NAR) and Previous Access Router (PAR).Also while using the nesting structure the top level router named by root mobile router (rMR) and the nesting routers named (nMR) as shown in Fig. 2. MNN A mobile node is a node can changes its location (Roaming among different subnets) within the Internet topology. $\mathrm{CN}$ is any node that communicates with the any MNN. HA is a router located usually in the home network of MNN that acts on behalf of the mobile node while away from the home link (http://en.wikipedia.org/wiki/Mobile_IPv6, surfed at 22/Aug/2010). When the MNN leaves its home subnet boarders, it notifies it's HA on its home link. The HA creates a Mobile Binding (BU), which is an association between the home IP address and current Care of Address (CoA). An address that is assigned to the mobile node when located in a foreign link. This address is based on the Prefix of the foreign link combined with the mobile node's interface identifier. After that there are two ways of sending and receiving the data between MNN and CN either by bidirectional tunneling or route optimization.

Correspond Author: Samer Sami Hasan, Computer Science, University Kebangsaan, Bangi, 43600, Malaysia 


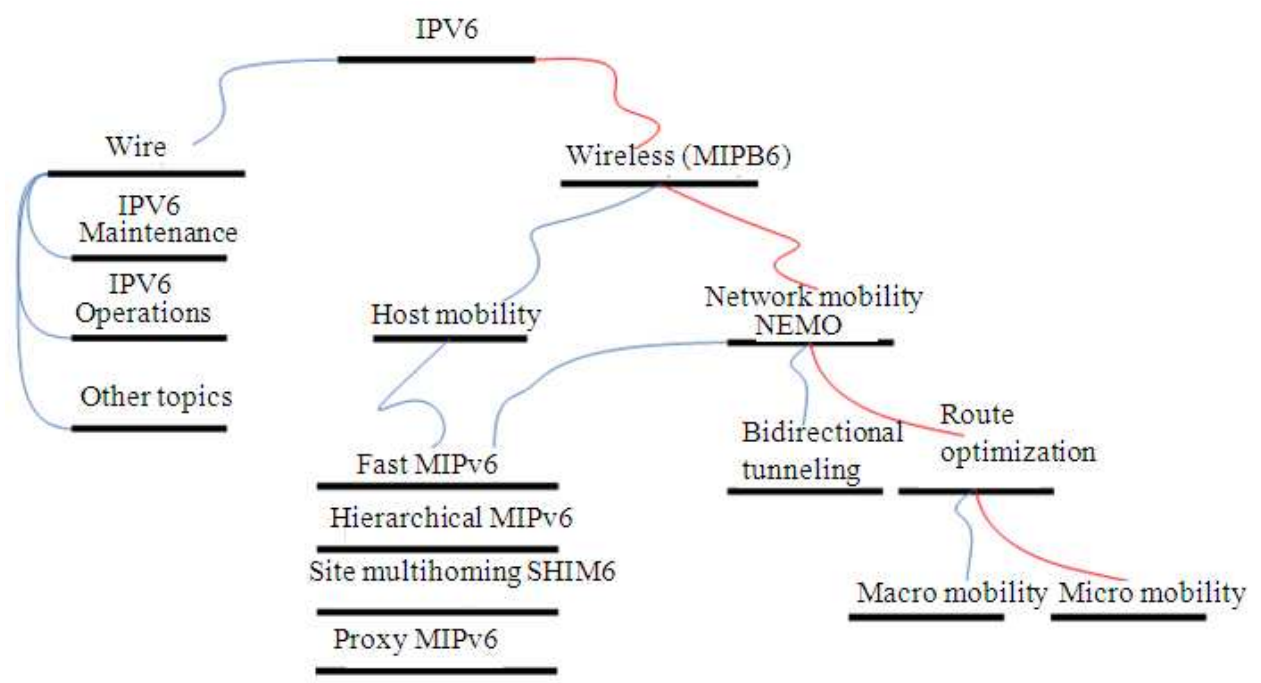

Fig. 1: Research directions on IPv6

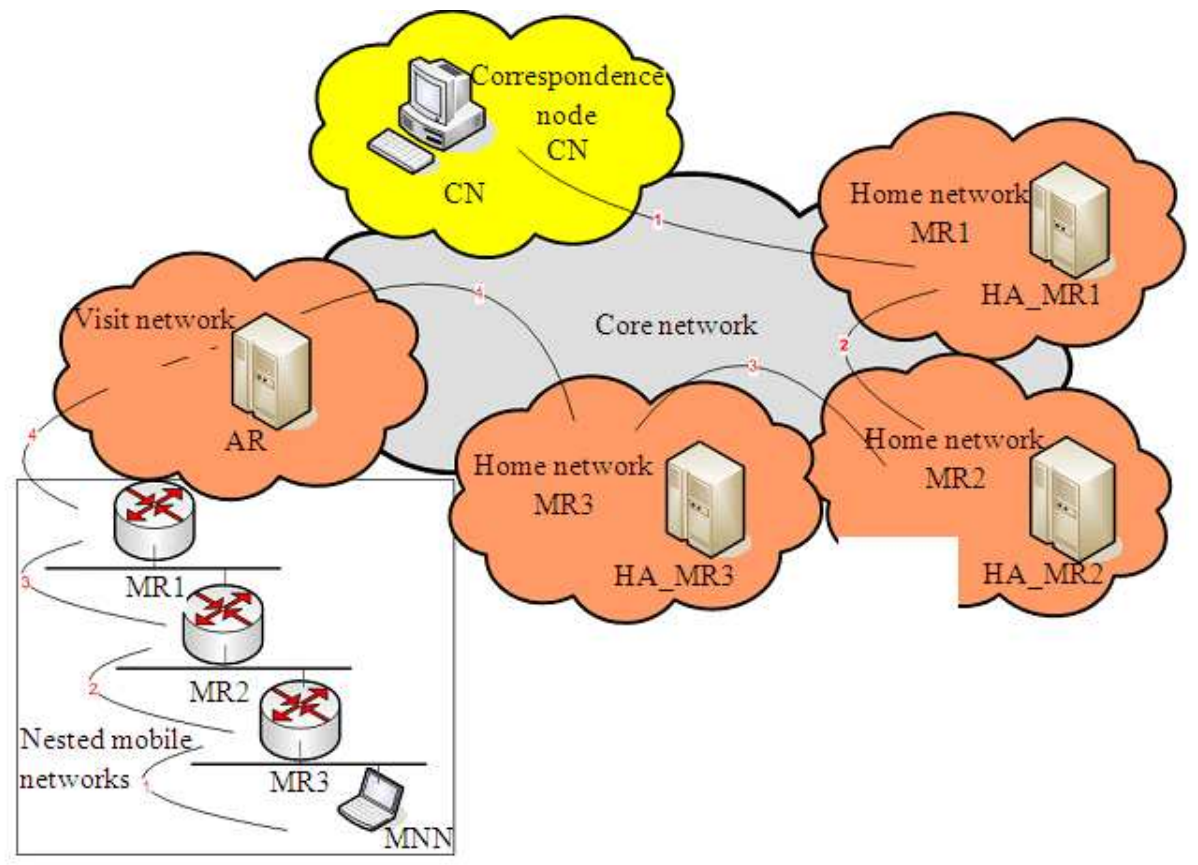

Fig. 2: NEMO basic entities

Bidirectional tunneling: In bidirectional tunneling the $\mathrm{CN}$ not supported by MIPv6, so the movement of MNN is transparent to the $\mathrm{CN}$. And the HoA is always used in communication at all times. Where the packets sends from the MNN to the CN it's always convoyed through the HA of the MNN, where imposing the information need into the header and by using tunneling to reach the actual location of MNN. On the other side the packets send from $\mathrm{CN} \rightarrow \mathrm{MNN}$ it will be tunneled back through HA by adding the additional information to the header (like: the CoA of MNN and the HA address) for routing the mobile network.

Route optimization: In Route Optimization the CN need to utilize the MIPv6 protocol. In the situation of MNN moves from the home link to the foreign link 
network the binding update request not only update the $\mathrm{HA}$ of the MNN but also the $\mathrm{CN}$ where the MNN wants to made a connection with it. So while the $\mathrm{CN}$ has all the information about the MNN and its new CoA then it will has the ability to route directly from $\mathrm{MNN} \rightarrow \mathrm{CN}$ without tunneling through the HA; therefore, a new header type generated to carries the additional information in addition of the original address of source and destination. When a $\mathrm{CN}$ wants to send a packet to specific IPv6 address, first it will check its binding cache if it have any index to this IP address. If exist it will be send the data to the appropriate CoA using the new routing header options. To the new routing header type it added to HoA of MNN. After receiving the data MNN sets the address destinated node (currently set to $\mathrm{CoA}$ ) to HoA it gets from the routing header option generated for $\mathrm{RO}$ to make the route optimization transparent to upper layers. When a $\mathrm{MNN} \rightarrow \mathrm{CN}$, it added its HoA to the new header. Then the $\mathrm{CN}$ that receives the data regenerate the source address (set to CoA) with the HoA. This assures that the application running at $\mathrm{CN}$ does not get any information about MNN's movement and its location (location transparency).

Route optimization schemes follow different approaches to increase the efficiency and the performance of network mobility, the next section show how various schemes that have been proposed can be worked:

\section{Route Optimized Tree Information Option} (ROTIO): In the ROTIO published by Cho Et Al. (Ng et al., 2007), where the rMR HoA and CoA of the nesting MRs(i.e. Routers between the rMR and MNN) are delegated to the nMR by using RA messages where the RA options contains the information of tree in the nesting structure. Each nMR add it's CoA to the convoyed RA which sent by the rMR then it will sends the RA reach down to the other nMRs level. Now each nMR Knows the CoA of the nMR from the Tree Information Option (TIO at the RA), so the MR sends two Binding Update messages: the First one binding the own home Agent with HoA of the rMR and the second binding is to the rMR with list of nMR CoAs exist above its tree. Therefore, each nMR can be reached and keep tracking on it through the HA of the nMR, rMR and its HA also, so the HA of an nMR having the ability to tunneling payloads to the HoA rMR's, then the rMR can be route payloads inside the network mobility depending on his knowledge about the nesting structure of network through the BU's. This proposed way having a disadvantage by increasing the tunneling level (i.e. nMR's HA $\rightarrow$ rMR's HA and rMR's HA $\rightarrow$ rMR). But also in the other side ROTIO compensated it by the lower signaling with easy to be deployed.

A Modified ROTIO for Nested Network Mobility (ROTIO++): in this scheme the Tree Information Option (TIO) used by ROTIO is extended to (xTIO at the RA) by Sircar et al. (2010), so the XTIO option will having the CoAs of the nMRs in a nesting NEMO, additionally, xTIO is upgraded at each BU message to inform an nMR of all its ancestor nMRs. The rMR makes the RA message with its rHoA in the Tree ID field and each nMR appends its CoA by using the extended tree information option. This scheme improve the ROTIO header overhead it becomes light than the ROTIO and with best degree of route optimization (end-to-end route optimization).Also no additional entities added to the main structure which means easy to be deployed and the movement of nMRs within the same rMR subnet there is no need to be BU with its HAs, so its reduces a significant signaling.

Host Identity Protocol (HIP): the HIP is proposed by (Moskowitz and Nikander , 2006), each mobile node uses a private address at the upper layers and the lower layers or the HIP are in a transparent manner to manage the positions changes. The first step in HIP communication each MNN generate a key that used for any update in locations. The basic idea of the HIP NEMO by using the key to authorize nMR to perform MNN locations update. When a MNN joins to the mobile network, the authorization takes its place. While increasing the nesting in NEMO the authorization is submitted at various levels. Each nMR uses the translation of prefix of the source address to a void tunneling while a packet sent from $\mathrm{MNN} \rightarrow \mathrm{CN}$. In this scheme like the other schemes the performance location management performed by rMR. The main disadvantage of this scheme is not easy to deployment with in the requirement of HIP, also this scheme provide optimal degree in end-to-end optimization, with out any tunneling at the cost of high signaling.

Optimized NEMO (ONEMO): this scheme published by Watari et al. (2006) in order to support nested NEMO (Devarapalli et al., 2005) scenario for enhancing the packet delivery, this scheme use new forwarding algorithm, signaling mechanism. The RA and BU technique in this scheme is complex to be deployable because of the new entities and functions added to the infrastructure like the Corresponding Router (CR) and Binding Proxy Agent (BPA) and also is using accompanied by CR Discovery technique and 
complicated MIpv6 binding mechanism, ONEMO costing additional signaling to reach near optimal route optimization.

MIPv6 Route Optimization for NEMO (MIRON): on the other hand the MIRON scheme proposed by Bernardo, et al. (2008) use the MIPv6 amongst the Return Routability (RR) procedure (Johnson et al., 2004) for RO but its not highly improve the NEMO RO only avoids the last tunnel between the nMR and it's corresponding and this scheme not handling nest topologies. The MIRON Caldaror et al. (2006) propose new technique for handling the nesting structure but this improving achieved by supplementing the base MIRON (Bernardo, et al 2008) with the third party to obtain a new CoA like PANA protocol (Jayaraman et al., 2008) and DHCPv6 (Droms, et al., 2003). This imposing making the MIRON more complex in the MR and the visited MNN, more complicated to be deployed.

Route Optimization Scheme for Nested Mobile Networks (NERON): this scheme proposed by Faqir et al. (2009), in this scheme each visited MR determines the address of the rMR's which is egress interface and its position inside the nesting subnet. Where the address and the depth of each MR are convoyed through the nesting level by adding new additional option specified by flag (R-flag), these option the Mobile Network Gateway Option (MNG) which contains are the rMR address and the Depth of each nMR, so each time the Nest Gateway Table (NGT) are updated through the MNG in RA convoyed in nesting structure. Now each MR receives a packet from its $\mathrm{MNN}$ destinated to $\mathrm{CN}$, it will first replace IPv6 header source address with the new CoA assigned from the egress interface; also add the address of the MNN in the home address destination option. All the nesting nMR by the way of the rMR will easily forward this packet until it reach the rMR, where it will be then forwarded the packets directly to the $\mathrm{CN}$, so the packet reach normally without encapsulation and direct RO by passing HA. The NERON solution is light weight signaling in comparing with MIRON and the performance of NERON with dependents of the depth and packets have zero tunneling overhead while exchange.

A Hierarchical Route Optimization (HROS): this proposed scheme by Gao $\mathrm{T}$. for next mobile network generation (Gao et al., 2008). This scheme use a new functional list MNN-CN list that's maintains the list dynamically through communication between $\mathrm{CN}$ and MNN runs through an optimal route during the movement of mobile network's. Where the packets are routed to nMR's current CoA nMR, no need to be intercepted by HA, which means its eliminate the pinball routing problem. In this scheme all of the MR will acts as Mobility Anchor Point (MAP), also there is no modifications to the other entities, made it easy to be deployable. Beside the RO, the HROS scheme it will reduce the time of packets encapsulation (only one encapsulation between $\mathrm{CN} \rightarrow \mathrm{MR}$ ), network resource is consequently saved, also the combination between NBS and MAP introduced by HIMPv6 (Castelluccia and El Malki, 2005) reduce the signaling between MR $\rightarrow$ HA.

\section{Localized Route Optimization Scheme for NEMO} (LROS): the localized scheme proposed by (Gao and Guo, 2009), an New Binding List (NBL) is generated to perform route optimization where each $\mathrm{MR}$ records the binding relation in its NBL between the $\mathrm{MNN} \rightarrow \mathrm{CN}$, so when mobile network move to the foreign network, the LORS scheme will optimized the route according to the new movements like HROS scheme, but when mobile networks returns to its previous home link through the NBL will be determined and the special operations of LORS scheme will be ignored and return back to the common MIPv6 network protocol. The LORS will reduce the cost of mobility management, while the packet over head comparing with NBS it has lower packet overhead than NBS within the large number of visited MNN.

Issues in route optimization: By specifying the main challenged reported from the literature works to perform route optimization between the $\mathrm{CN} \rightarrow \mathrm{MNN}$, several issues raises in addition of the packet header over head as following:

- Signaling: Which means the competition of signaling packets with the payload (data packet)

- Packet header overhead: The information added to the main header for RO

- Degree of Deploybility: The new functions and entities added to the base infrastructure of MIPv6

- End-to-End RO: Which means the degree of RO and the consuming load on the infrastructure

- Intra-NEMO: The RO between two MNN with in the same network mobility

Depends on the Criteria (derived above), Table 1 presents a comparative summary of the schemes based on either the analytical models or the simulation experiments tested by each scheme separately for the above issues. After comparing these schemes wither if it's topologically correct or incorrect we find that there is no suitable scheme which is suits all mobility scenarios. 
Am. J. Applied Sci., 8 (6): 579-583, 2011

Table 1: Comparison between route optimization schemes

\begin{tabular}{|c|c|c|c|c|c|c|c|c|}
\hline & ROTIO & ROTIO++ & HIP & ONEMO & MIRON & NERON & HROS & LROS \\
\hline Signaling & Moderate & Good & Heavy & Moderate & Heavy & Moderate & Moderate & Moderate \\
\hline $\begin{array}{l}\text { Packet header } \\
\text { overhead }\end{array}$ & Moderate & Light & Moderate & Heavy & Light & Moderate & Moderate & Light \\
\hline $\begin{array}{l}\text { Degree of } \\
\text { deployability }\end{array}$ & Easy & Easy & Difficult & Difficult & Difficult & Easy & Easy & Easy \\
\hline End-to-End RO & Moderate & Moderate $^{+}$ & Good & Good & Good & Good & Moderate & Moderate \\
\hline Intra-NEMO & Good & Good & Poor & Good & Poor & Moderate & Poor & Poor \\
\hline
\end{tabular}

\section{CONCLUSION}

One of the most important and emerging topic is the routing optimization in nested mobile scenarios. At the end point of view an end-to-end delay increased by inefficient route, resulting the performance in realtime trends to be in degradation. So the problem further increased with increasing of nesting level. Nowadays most of the research efforts the concentrate on Route Optimization of NEMO, like solving the problem of header overhead and inefficient route for mobile network. This study, study the state of art the RO NEMO schemes and made an comparison between various schemes in its the adaptation ability to the infrastructure of the Internet. The comparisons based on different criteria to supports an efficiency of mobile network.

\section{REFERENCES}

Bernardo, C., 2008. MIPv6 Route Optimization for Network Mobility (MIRON)'. Internet Draft, IETF, (cjbc@it.uc3m.es).

Calderon, M. et al., 2006. MIRON: Mobile IPv6 route optimization for NEMO. IEEE, DOI: 10.1109/JSAC.2006.875109.

Castelluccia, C. and K. El Malki, 2005. RFC4140: Hierarchical mobile IPv6 mobility management. ID Tag: draft-ietf-mipshop-hmipv6-04.txt, (h.soliman@flarion.com), tools.ietf.org/pdf/rfc4140.pdf .

Devarapalli, V., R. Wakikawa, A. Petrescu and P. Thubert. 2005. RFC 3963 NEtwork MObility (NEMO) basic support protocol, IETF, NEMO Working Group, http://www.ietf.org/rfc/rfc3963.txt

Droms, R. et al, 2003. RFC 3315: Dynamic host configuration protocol for IPv6 (DHCPv6), IETF, Networking Group, http://www.ietf.org/rfc/rfc3315.txt

Yousaf, F.Z.; Tigyo, A.; Wietfeld, C.; 2009. NERON: A route optimization scheme for nested. 978-14244-2948, IEEE, DOI: 10.1109/WCNC.2009.4917826
Gao T. and N. Guo, 2008. LROS: Localized route optimization scheme for NEMO', Software College, Northeastern University, Shenyang, China, Gaoth@mail.neu.edu.cn

Guonan@ise.neu.edu.cn,

DOI: 10.1109/TENCON.2008.4766613

Gao Han, 2008, A hierarchical route optimization scheme for next generation mobile network. IEEE, DOI: 10.1109/IWCMC.2008.15

http://en.wikipedia.org/wiki/Mobile_IPv6, surfed at 22/Aug/2010.

Jayaraman, P. et al., 2008. RFC5193: Protocol for Carrying Authentication for Network Access (PANA) Framework, IETF, (prakash_jayaraman@net.com)http://www.otaroo.n et/ietf/rfc/rfc5193.txt.

Johnson, D., C.E. Perkins and J. Arkko, 2004. RFC 3775: Mobility support in IPv6. IETF, (dbj@cs.rice.edu ), tools.ietf.org/pdf/rfc3775.pdf .

Moskowitz, R. and P. Nikander. 2006. RFC 4423: Host Identity Protocol (HIP) architecture. IETF, (rgm@icsalabs.com), tools.ietf.org/pdf/rfc4423.pdf

Ng, C., P. Thubert, M.Watari and F. Zhao, 2007. RFC 4888: Network mobility route optimization problem statement. IETF, (chanwah.ng@sg.panasonic.com), tools.ietf.org/pdf/rfc4888.pdf .

Sircar, A., B. Sardar and D. Saha, 2010. ROTIO+: A Modified ROTIO for Nested Network Mobility. IT Dept, Jadavpur University, Kolkata, ansuman.sircar@tcs.com, bhaskargit@yahoo.co.in, Kolkatads@iimcal.ac.in.

Watari, M., T. Ernst and J. Murai. 2006. Routing optimization for nested mobile networks'. IEICE Trans. Commun., vol E89-B: 2786-2793. online ISSN: 1745-1345, ( watari@kddilabs.jp

Zafar, A., M. Shahriar and M. Atiquzzaman, 2010. Senior Member. IEEE and William Ivancic, 'route optimization in network mobility: Solutions, classification, comparison and future research directions. IEEE Communications Surveys and Tutorials, 12, First Quarter. DOI: 10.1109/SURV.2010.020110.00087 